\title{
4th symposium on primary breast cancer in older women. Theme: putting personalized care into practice (Held: 3 March 2017)
}

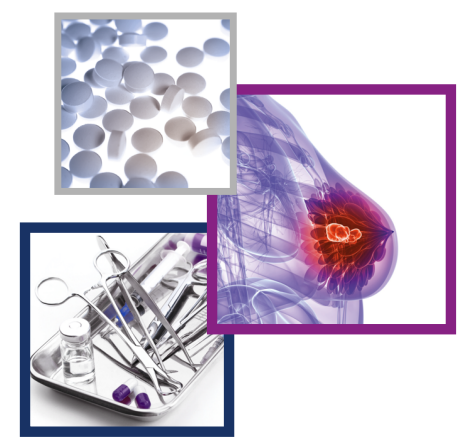

\author{
Kwok-Leung Cheung*,1, David Morgan², Etienne Brain ${ }^{3}$, Philip Poortmans ${ }^{3}$, Ruth Parks ${ }^{4}$, \\ Beatriz Korc-Grodzicki ${ }^{5}$, Fiammetta Ugolini ${ }^{6}$, Taner Shakir ${ }^{7}$, Janice Tsang ${ }^{8}$, Heather Stone ${ }^{9}$, \\ Cindy Kenis ${ }^{10}$, Graeme Perks ${ }^{2}$ \& Roshaine Wijayatunga ${ }^{11}$ \\ ${ }^{1}$ Division of Medical Sciences and Graduate Entry Medicine, School of Medicine, University of Nottingham, Royal Derby Hospital \\ Centre, Derby DE22 3DT, UK \\ ${ }^{2}$ Department of Oncology, Nottingham University Hospitals, Nottingham NG5 1PB, UK \\ ${ }^{3}$ Department of Oncology, Institut Curie, Paris, France \\ ${ }^{4}$ Breast Unit, Royal Derby Hospital, Derby DE22 3NE, UK \\ ${ }^{5}$ Geriatrics Service, Memorial Sloan Kettering Cancer Center, New York, NY 10065, USA \\ ${ }^{6}$ Breast Unit, Brighton \& Sussex University Hospital, Brighton BN1 6AG, UK \\ ${ }^{7}$ Peterborough Breast Unit, Peterborough PE3 9GZ, UK \\ ${ }^{8}$ Hong Kong Breast Cancer Registry, Hong Kong Breast Cancer Foundation, Hong Kong, SAR \\ ${ }^{9}$ Breast Unit, Royal Derby Hospital, Derby DE22 3NE, UK \\ ${ }^{10}$ Department of Geriatric Oncology, University Hospitals Leuven, Belgium \\ ${ }^{11}$ Future Science Group, Unitec House 2 Albert Place, Albert PI, London N3 1QB, UK \\ * Author for correspondence: kl.cheung@nottingham.ac.uk
}

Following the inception in 2010, the University of Nottingham hosted the 4th Symposium on Primary Breast Cancer in Older Women, under the auspices of the International Society of Geriatric Oncology, in March 2017, at East Midlands Conference Centre. This is the only meeting of its kind in the UK, now held biennially, aiming at a multidisciplinary audience, including patients, their carers and advocates. With a theme on 'Putting personalising care into practice', this Symposium included sessions on 'local and systemic therapies', 'new ideas', 'patients and carers', and 'challenging areas', covered by an international and local faculty, interviewing patients and carers, and abstract presentations. Topics covered were practical and wide-ranging, including selectng for chemotherapy, radiotherapy and breast reconstruction, treating HER2-positive disease, and the roles of the geriatrician and geriatric oncology nurse.

\section{Keywords: breast cancer $\bullet$ geriatric oncology $\bullet$ older women $\bullet$ primary breast cancer $\bullet$ symposium}

The symposium began with a welcome and introduction from Kwok-Leung Cheung (Clinical Associate Professor, University of Nottingham, Consultant Breast Surgeon, Royal Derby Hospital; UK National Representative, International Society of Geriatric Oncology [SIOG], Symposium co-chair). Cheung began by extending a warm welcome to everyone to the East Midlands Conference Centre and the University of Nottingham. He explained how the symposium, first started in 2010 , began in a very small room in the postgraduate education center in City Hospital, Nottingham, with only about 20-30 people in attendance. He was pleased to say that things had certainly moved on, and the biennial symposium was now a multidisciplinary event with an emphasis on patient perspectives, as a direct response to delegate feedback ranking patient sessions the best!

The symposium has continuous professional development accreditation from the Royal Colleges and is basically the only event in the country to be dedicated to a multidisciplinary focus on older women with primary breast cancer. Cheung was also happy to announce that since the event is under the auspices of SIOG, senior members of SIOG will be speaking and presenting also.

Since the previous symposium, organizers have been giving a theme to the event, this year's theme being, 'Putting personalized care into practice', with an emphasis on the word 'personalizing', since it is well known that older women with primary breast cancer tend to be under- or overtreated sometimes due to a lack of knowledge and other times due to over enthusiasm or failure to include them in clinical trials. 
Also based on requests and feedback from previous conferences, Cheung explained the addition of a 'students and patient advocates' category at registration for the conference to encourage their attendance.

The speaker list went beyond just oncological representations of surgeons and oncologists, including an epidemiologist and a geriatrician from the previous symposium and in the 2015 event, the geriatrician being particularly inspiring - especially for the UK delegates, since it is not really a standard practice in the UK to include a geriatrician in the care of older women with primary breast cancer. For the first time, there was a geriatric oncology nurse in the faculty - Cheung termed this a 'new breed' or 'new concept' to the UK, since although we have dedicated specialist nurses to specific tumor sites, for example, breast care nurses, colorectal care nurses and so on, geriatric oncology nurses are almost unheard of in this country.

In addition to a presentation on the topic of breast reconstruction in older women, to be delivered during the symposium by a distinguished plastic and reconstructive surgeon, oral presentations were also added to the program so that speakers could share their specific experiences. Furthermore, the organizing committee this year was expanded to include a surgical trainee.

The symposium abstracts and program has now been published in Future Oncology as an online supplement [1] and the slides from the presentation are available on the symposium website [2].

Cheung ended his introduction by thanking the sponsors of the event and encouraging delegates to visit the stands during the break times and also to take time to look at the poster displays. He encouraged people to join SIOG and directed them to the SIOG website for further information [3].

\section{Session 1: local \& systemic therapies}

The first session on 'Local and systemic therapies' was chaired by David Morgan (symposium co-chair) and consisted of two presentations followed by a discussion session.

Dr Morgan began by also extending a welcome to everyone and was happy to say that he believed it was one of the best turnouts since the symposium first began. He explained that in this first session, they would be focusing on nonsurgical treatments - chemotherapy and radiation. One of the 'problems' with older patients is finding the right balance, so as not to under- or over-treat them. Older patients by definition are more likely to suffer from diseases associated with age, and so frailty as a general concept afflicts older patients more often than younger ones, therefore it is important to find the correct balance - requiring more 'personalization', personalization being the keyword for today.

\section{Selecting for chemotherapy: the use of genomics}

The first talk in the session was given by Etienne Brain, Medical Oncologist at the Institut Curie in France and also the Immediate Past President of SIOG, on the topic of "Selecting for chemotherapy - the use of genomics".

Dr Brain explained that although there have been great strides in the past decade in cancer management in general, this has not benefited the entire population, with older persons deriving less benefit overall. The main dilemma faced when approaching any cancer in the older patient is ageism - when you decide not to treat as you consider the patient is too old - referred to as 'therapeutic nihilism', and the opposite also can be true 'blind therapeutic enthusiasm' - the use of new treatment innovations bringing futile benefits with potential and uncontrolled side effects.

The stark differences in the biology of breast cancer was displayed on one slide $[4,5]$. According to standard pathology reports, more luminal cases are seen in older women; on the molecular levels it is apparent that less aggressive or basal-type cases are also seen in older women. Dr Brain stressed that the difference actually appears not above 70 or 75 years of age, but rather much earlier, after a postmenopausal setting.

Quite often therefore, these patients are undertreated and it happens on a very regular basis and delegates were shown a graph demonstrating that the use of chemotherapy for node-positive $(\mathrm{N}+)$ cases (estrogen receptor-positive $[\mathrm{ER}+] \mathrm{N}+$ stage I/II breast cancer) decreases dramatically according to age [6], mirroring the fear regarding side effects of chemotherapy, leading the physician to not take the risk of delivering such treatment in the adjuvant setting. Underusing can lead to undertreatment and in turn lead to a worse prognosis in these patients. From the same series and others [6,7] we were shown that breast cancer mortality increases by $20-30 \%$ in older patients compared with their younger counterparts. It is important to note that mortality due to other causes increased even more with age therefore a proper balance is required in the decision-making regarding the treatment process.

The converse to this is overtreatment - a sizeable proportion of older patients with operable breast cancer die of noncancer-related causes and therefore absolute benefit of treatments is lower. This presents the main challenge 
when making these kinds of decisions. Adjuvant chemotherapy can work irrespective of age and the difference arises in the side effects and mortality rate being far higher than what you see in younger adults [8]. When you look at big series with standard pathologic characteristics, chemotherapy is mostly useful in ER cases [9,10], demonstrating that biology drives the potential benefit of preventative treatment delivered.

Algorithms used to make decisions in the adjuvant setting are very inadequate and some have been shown to be very badly developed for the older population. For example, 'Adjuvant! Online' is the most achieved tool that is used in clinical practice; however, it is not accurate in older patients, nor it is accurate in very young patients as it was developed in a standard adult population. 'Predict' is another algorithm based on standard characteristics for the tumor, but predicts only for overall survival not taking into account comorbidities and does not give any information on the risk of relapse very well [11,12].

Gene expression profiling was developed since the early 2000s to identify prognosis and also to identify patients requiring chemotherapy. The other pending question that came up from this was how we can then avoid chemotherapy in some cases. All this was for the development of better individual risk stratification [13].

There are around six different tools available - some still in development, and all based on the quantification of nucleic acid using different techniques. They do not all provide the same information and have been developed in specific settings. These tools provide the continuous variable or sometimes they dichotomize the population. A very important point stressed to us at this point was that some tools are centralized in terms of determination while others can be performed in a decentralized way. Furthermore, it should be noted that most of the trials did not include women of $65+/ 70+$ years of age.

Dr Brain went on to explain a few tools in detail. MammaPrint brings some prognostic information to the table, allowing the de-escalation of the indication of chemotherapy. This tool was investigated in the MINDACT program [14] and since none of the patients were $>70$ years of age, it gives a skewed picture. MammaPrint provides information on the risk of distant recurrence at 5 years without any treatment and this gives general results. How this can be extrapolated or utilized in the older population remains questionable.

Another tool, OncotypeDX is different. This tool assesses the risk of distant recurrence at 10 years with the use of tamoxifen for 5 years in node-negative patients; and 5 years with the use of tamoxifen for 5 years in $\mathrm{N}+$ patients. Evaluation can therefore be confusing since the end points and conditions are different. However, it does give personal results and that is an important difference compared with MammaPrint and it has been investigated specifically in the older population, based on a large dataset derived with collaboration with the NCI and Genomic Health, presented by S Shak at the last European Society for Medical Oncology (ESMO) conference [15]. The work specifically focused on the use of the tool on node-negative $(\mathrm{N}-)$, hormone receptor-positive (HR+), HER2negative cases and looked at the use of this innovation in older populations, selecting almost 250,000 cases. Some of these cases were tested with the recurrence score and others had not been. Looking at the partition between the $70+$ and $<70$-year-old patients, there was about three-times less testing of and use of recurrence score in the older patients. Chemotherapy use was lower in patients $\geq 70$ years of age tested or untested.

As Dr Brain explained, what was important is that the use of the recurrence score according to age shows that above or below 70 years of age, it provides some prognostic information above a recurrence score of 18 . Recurrence score predicts breast cancer-specific mortality in both age groups. Higher other-cause mortality was observed in older patients and notably, 5-year breast cancer-specific mortality is relatively high in untested patients of all ages, therefore deserving further study.

Dr Brain explained MammaPrint used in ASTER 70s [16], an ongoing study, with about 2000 patients above 70 years of age in the adjuvant setting after chemotherapy or after relapse in very specific settings.

When all these signatures are compared, Dr Brain explained that many overlaps can be seen. A prospective study was conducted [17] that assessed the value of different signatures and this showed that all brought some kind of prognostic information but the overlap in the genes assessed is not complete and there are some exclusions. These tools do not always agree even if the assessments are refined, since although the risk estimates are close at the population level, there are individual differences.

He stated that the crux in the older population is the competing risks of mortality that exists in breast cancer and also other causes of mortality that can arise. It is therefore not so much about two worlds confronting one another (there is a feeling that oncology cannot work very well with geriatrics) but very often it is a question of sharing the information. Molecular signatures and gene expression profiles are just as important as the information that is brought from the comprehensive geriatric assessment. Dr Brain concluded with the importance of working together - which is the basis of geriatric oncology. 


\section{Selecting for radiation therapy}

Dr Brain's talk was followed by a presentation given by Philip Poortmans, Professor of Radiation Oncology, until very recently Radboud University, The Netherlands (since 1 March Institut Curie, Paris, France), Past-President of the European Society for Radiotherapy and Oncology and President-Elect of the European Cancer Organisation, on the topic of 'Selecting for radiation therapy'.

Poortmans began by talking about life expectancy. Life expectancy in years has increased with Australia having been in the lead for the past several years. Expectations have been beaten continually - the line on the life expectancy graph continues to go up [18]. This fact holds a lot of importance for the patients focused on in this symposium. How do you define an 'old patient'? What is their life expectancy? Poortmans highlighted that life expectancy at birth is very different to the actual life expectancy that an individual has later on in life.

Although in England and Wales, a woman aged 65 is expected to reach roughly around the age of 93, we are still not doing too well - since for patients above the age of 70 years, the relative survival up to 10 years after diagnosis is decreasing [19]. There has been a little improvement in this over the last few decades, but there is still quite some way to go.

We were then asked - "what is the role of radiation therapy in general in breast cancer?" After breast conserving therapy, the effect of radiation therapy in patients with no involved axillary lymph nodes results in a 'any first recurrence' rate at 10 years decrease by $15.4 \%$ with a slight $3.3 \%$ influence on breast cancer-related mortality at 15 years. For every five 'any recurrences' prevented at 10 years, you have one breast cancer-related death less at 15 years [20].

Poortmans then displayed data showing that if you take patients with involved lymph nodes, the effect is slightly higher $-21.2 \%$, and the effect on breast cancer-related mortality is more impressive with $8.5 \%$. Thereby, here only 2.5 'any recurrences' need to be prevented with radiation therapy to have one breast cancer-related death less at 15 years [20].

Many trials tested whether radiation therapy is required after tumorectomy in 'low-risk' older patients, but the criteria for inclusion differed from younger age of 50 up to at least 65 years of age depending on the trial. Poortmans focused on just the BASO II trials, as in his opinion it gives a good insight as it had four trial arms: no treatment arm (so patients had no radiation therapy, no endocrine therapy or no systemic therapy in general) after lumpectomy; radiation therapy arm; systemic therapy arm; and both treatments arm [21]. The results are clear. If no treatment at all is given, the recurrence rate at 10 years is about $20 \%$. If radiation or tamoxifen is given, the recurrence rate drops to about $3 \%$ and both treatments together resulted in only one recurrence (therefore local control rate was $99 \%)$.

After working $>25$ years in The Netherlands, Poortmans explained that virtually none of the patients in the above trial would get adjuvant systemic treatment according to the Dutch Guidelines. The Netherlands is one of those countries in which $>20 \%$ of patients do not receive anything thank to their low recurrences risks and the thereby limited benefit of systemic treatments that is outweighed by the side effects.

Poortmans posed the question - "So is radiation therapy after tumorectomy always required?" Generally, he said that he did not think it is required for all patients. In the past we accepted $1 \%$ local recurrences per year after treatment but this has gradually decreased to a maximum of $0.5 \%$ per year. It is important to keep in mind late relapses, and also to remember that second primary tumors can arise in both the treated and the contralateral breast. It is also important to consider the role of systemic treatment in this.

Poortmans then described to us a very interesting and specific case to illustrate that every patient is unique and treatment really needs to be individualized.

Radiation therapy has improved a lot. There are several ways for this with opinions differing across the USA and Europe, for example [22]. Poortmans proposed that we should urgently stop thinking in terms of 'treatment fields' but instead think of 'target volumes' - requiring prescriptions including dose objectives aside constraints for organs at risk.

Respiratory control - now being widely introduced in the UK - is standard in The Netherlands for all left-sided patients and some right-sided patients. It is not there to decrease the movement of the patient, but rather to increase the separation between the target and the heart so that the dose which is 'accidentally' given to the heart can be decreased and the risk of complications lowered.

In a 2017 publication in Breast [23], it was demonstrated that breast conserving therapy rates had improved. In an early pilot trial in the eighties, comparing breast conserving therapy with mastectomy, at 10 years the local recurrence 
rate after breast conserving therapy was nearly $20 \%$. This dropped to about $10 \%$ at 10 years in the European Organisation for Research and Treatment of Cancer (EORTC) Boost no Boost Trial of the nineties. A recent analysis done in the Young Boost Trial (2004-2011) [24] shows that there are only 1.8\% locoregional recurrences at 9 years in patients $<51$ ! We were then presented with confirmatory data from more than 37,000 patients from the Dutch population-based cancer registry [25].

Guidelines regarding partial breast irradiation have been published from Europe [26] and the USA [27]. At the same time, whole breast irradiation is evolving and in the UK FAST-Forward trial (not yet published), the conventional UK schedule of 40 Gy and 15 fractions in 3 weeks is compared with 27 Gy and 26 Gy in 5 fractions in just 1 week.

Poortmans explained that we need to look beyond technical improvements. We also have signatures from a molecular and genomic base - for example, the wound model has been tested in breast, lung and gastric cancer $[28,29]$. In one trial testing this, high-risk patients had a higher rate of local recurrences in early breast cancer compared with the patients with the low-risk signature [30].

We were shown a slide demonstrating how the side effects associated with radiation therapy in the past have now improved - for example, less inconvenience due to shorter treatment times, less skin toxicity among others.

In his conclusion Poortmans explained that life expectancy should play a very important role in our decisionmaking process. We also need to define the tumor risk. The next important step is shared decision-making, including a very open discussion with patients. We were presented with various scenarios for treatment - for example, for early stage, low-risk patients with a short life expectancy you would perhaps consider either surgery alone, endocrine therapy alone or maybe nothing at all, whereas for early stage, low-risk patients with long life expectancy, you could consider both surgery and radiation therapy.

Finally, Poortmans described how from 40 years ago to now, the relative mortality incidence has decreased from one out of 2.5 (for every 2.5 patients diagnosed at the same time, one died) to one out of six (for every six patients diagnosed, only one died) - this is a huge improvement. In his opinion, de-escalating is a good thing but he suggested that it should be performed in a stepwise manner. At present, the medical community has quite a lot of trust overall from patients, and once that trust is lost, a lot of work will need to be done to restore it once again, therefore it is important to avoid making big mistakes.

The session on 'Local and systemic therapies' ended with the session chair opening up the floor for questions to both speakers and after a short discussion the program moved on to a coffee break and poster viewing.

\section{Session 2: 'new' ideas}

This next session was chaired by Ruth Parks and featured a presentation and discussion, along with the presentation of four oral abstracts.

\section{Geriatrician in the preoperative assessment clinic}

The first talk in this session was presented by Beatriz Korc-Grodzicki, Chief, Geriatrics Service, Memorial Sloan Kettering Cancer Center, New York, USA, on the topic of 'Geriatrician in the preoperative assessment clinic'.

The trend in preoperative risk assessment for the older breast cancer patient is to take in to account 'frailty'. Looking at the literature published between 1979 and 2016, under 'frailty' and 'mortality', the number of publications over time has increased dramatically. So what is 'frailty'? The concept of frailty in the population of older adults that are going to be diagnosed with cancer (breast cancer or otherwise) has varied over time and there have been multiple definitions of frailty.

Frailty is "a syndrome of advancing age characterized by immune dysregulation, chronic inflammation, sarcopenia, increased cellular senescence and a loss of resilience". In this definition, frailty is a syndrome of advancing age; however, not everybody that is frail is of an advanced age and many times it can be the cancer treatment that leads to developing frailty even though those patients are not part of the 'older population'. Korc-Grodzicki highlighted the most important characteristics of frailty (clinical features), namely, decreased functional reserve, impairment or dysregulation in multiple physiological symptoms and the reduced ability to regain physiological homeostasis after a stressful and destabilizing event.

We were told that frailty has been used for several years in the definition or implication of risk assessment for outcomes in surgical patients. In a study published in 2010, it was demonstrated that when frailty is added to risk indices such as the American Society of Anesthesiologists index, the Lee risk index or the Eagle risk index, the risk assessment for outcomes is improved [31]. 
Delegates were presented with the case of Miss A. She was advised to and decided to go for a screening mammogram. She is an 82-year-old patient with hypertension, diabetes and peripheral neuropathy with chronic kidney disease related to her diabetes. She can walk slowly for a few blocks and uses a cane. Her screening mammogram results in an abnormal mammogram and her final diagnosis was T1 and T2 breast cancer. She had a $1.5 \mathrm{~cm}$ mass with some positive lymph nodes. After biopsy she was noted to be triple negative and it was decided that she should have a lumpectomy and a lymph node dissection followed by adjuvant chemotherapy and radiation therapy in the future.

The first question to ask would be, "Will the patient tolerate and benefit from this treatment?" This question would probably need to have been asked before it was even suggested to the patient that she should have a mammogram, and should also be asked after her diagnosis and prior to making a treatment plan.

Korc-Grodzicki highlighted the fact that not everyone would be the same. You could have a wheelchair-bound 82-year-old gentleman, living in a nursing home with multiple comorbidities, multiple medications and completely functionally dependent, compared with an 82-year-old gentleman who has very little comorbidities, only takes one aspirin daily, is very fit and lively and is into a variety of different physical activities. It is fair to say that the 82-year-old gentleman in the nursing home will be frail and have a general poor tolerance to treatment, in comparison to the other gentleman who will probably have a tolerance to treatment similar to that of a middle-aged patient [32].

Miss A falls under the category of a 'vulnerable' patient - in the middle and not at either extreme. So it is necessary to have a better understanding of their individual situation in order to understand how they will tolerate treatment. Korc-Grodzicki explained that people fall into the risk of saying a patient is 'too old' so they will not tolerate treatment since that patient has multiple comorbidities and is not that mobile. However, many times, the age of the patient is in the eye of the beholder. This can depend on the age of the physician and also on the little bit of ageism that is around. This can lead to the risk of undertreatment and is a strong risk factor for a prognosis in older patients.

In a study published in 2003 [33] it was shown that older women with breast cancer generally have a later diagnosis, many times they can have an incomplete diagnosis assessment and they lack a standardized therapeutic approach. As many as $50 \%$ of them had suboptimal treatment, and this resulted in a large excess of preventable breast cancer.

The opposite can be true if one just focuses on the cancer and not the patient. There is a possibility of overtreatment and this can lead to unacceptable postoperative outcomes with high mortality or persistent disability [34].

Korc-Grodzicki stressed the importance of personalized assessment of the older patient, looking not just at the chronological age of the patient, but also the life expectancy of the patient and the functional reserve of the patient as they are at the moment of diagnosis. A nice approach to decision-making was published in the National Comprehensive Cancer Network (NCCN) Guidelines [35] - it goes into a series of questions to allow you to put things into perspective.

The first question is, "Is the patient at moderate or high risk of dying or suffering from cancer considering his or her overall life expectancy?"

For example, if a patient diagnosed with breast cancer has end-stage chronic obstructive pulmonary disease, then the breast cancer is probably not going to be a risk for that patient in terms of mortality or even suffering from the cancer. For this patient the answer to this first question will be 'no' and they will just need to be provided with symptom management and supportive care.

If the answer is 'yes', the next question to ask is, "does this patient have decision-making capacity?" Can the patient understand what the problem is? Can they understand the characteristics of treatment and the pros and cons? Can they understand what the different treatments will accomplish? Korc-Grodzicki said that in her clinic, the first question she asks to patients after saying "hello", "how are you?" and "pleased to meet you" is "please tell me in your own words what is the disease that you have and what is your understanding of the treatment being offered". The answers received can be very surprising. Many patients do understand and are very clear; however, some patients are so confused and cannot put the diagnosis and treatment together. This can be related to cognitive difficulties, but many times it is due to the fact that people become very anxious, explanations are very quick and they are scared of asking questions.

If the patient is unable to make their own decision, then it will be necessary to obtain information about this patient from the patient care giver, relatives or other physicians who know the patient well. 
If the patient is able to understand and make decisions with the geriatrician/surgeon/oncologist, then the next question to ask is, "are the patient's goals and values consistent with wanting anticancer therapy?"

Korc-Grodzicki described how some patients will acknowledge that there is cancer and it absolutely needs to come out. Others, however, will say that they have a wonderful life, and since the cancer is asymptomatic, and they want their independence and quality of life, they will return whenever symptoms come. If the answer is "yes, they do want the treatment to go ahead", the next step would be to assess for risk factors prior to going into the clinic for treatment.

Geriatricians assess risk factors using the 'Geriatric Comprehensive Assessment'. The definition of this has changed over time and is now, "a multidimensional, interdisciplinary patient evaluation that leads to the identification of patient problems and the development of a plan for resolving these problems". It is an assessment that will identify problems that are usually not identified with a history check and physical examination alone. When problems are identified, the goal is to try and improve these prior to getting the appropriate treatment.

The geriatric assessment includes multiple domains, namely:

- Function: is the patient able to take care of themselves, what is their performance status, gait and balance among others;

- Polypharmacy: how many prescription and nonprescription medications they take, how adherent they are to these medications (particularly important if a patient is going to be treated with oral medications for cancer) among others;

- Comorbidities;

- Cognition;

- Nutrition;

- Social support: living conditions, what is the care giver situation?

- Psychological state: the anxiety and the depression that comes with cancer diagnosis and treatments;

- Other geriatric syndromes: such as hearing loss or difficulties with vision. If a patient cannot hear and their physician is unaware of it, the patient will be unable to follow recommendations, or they may not be able to talk on the phone with a nurse when they are given a call. Vision impairment can mean they may not be able to read instructions they are given regarding their treatment or diagnosis.

In Korc-Grodzicki's presentation we heard that the geriatric focus is very much patient care. The patient is at the center and not the disease. The disease is just one more piece of the treatment puzzle. The importance of geriatric assessment in preoperative risk assessment has been widely published. Many papers can be found in the literature from over the last 10 years or so. One example is the paper by Robinson et al. [36] - examining 68-80-year-old patients who had elective surgery that required intensive care unit admission. What Robinson et al. discovered was that impaired cognition, recent falls, low albumin and low hemoglobin, as well as functional dependency and increased comorbidities are risk factors for 6-month mortality and postdischarge institutionalization. KorcGrodzicki mentioned that in her institution, they demonstrated that patients who are 75 years or older who have major cancer surgeries that require $>1$ day of hospitalization, when they have multimorbidity, functional dependency and a history of falls, have an increased risk for postoperative delirium, increased length of stay and discharge to rehabilitation [37].

Returning to the case of Miss A - we were told that she decided to have a mammogram and was referred to the clinic for geriatric assessment before going for breast cancer surgery. In addition to the details we had already heard about Miss A, we learned that she had two falls over the paclitaxel 6 months, a $4 \mathrm{lb}$ weight loss over the past 6 months (that is $10 \%$ of her weight) and she had been a smoker and had given up about 20 years prior. She lived with her husband of 50 years. Her husband has dementia and they have one child who lives 30 miles away. The child is involved but she has her own family, her own full-time job and sees her parents maybe once every 2 or 3 weeks. Miss A is actually the main carer for her husband. Her medication list is extensive in terms of prescription medications and over-the-counter medications. In her physical exam she was a very shy woman, exhibited no distress but had very poor eye contact, was very depressed. She had decreased pin-prick sensation consistent with her PN, otherwise was normal.

After the geriatric assessment was conducted on this patient, many things became apparent. In terms of her function - the patient was dependent in some of the basic activities of daily living. She could walk independently in the home, and one block outside. For anything further she needed to be with somebody and needed her cane. 
She required help for shopping, to prepare food, major housework and laundry. However, she managed her own extensive medications and also her husband's extensive medications. Her long medication list needed to be cleaned up and simplified.

After evaluation, unintentional weight loss and depression was added to her list of comorbidities. She was evaluated with Mini-Cog for cognition evaluation and this is a very short test and her result was abnormal meaning that she has an increased risk of dementia or the possibility of cognitive impairment. We were told that she had an abnormal clock drawing test and two out of three recalls.

Looking at the nutrition, social support and psychological state domains of the assessment, the weight loss the patient had was related not only to her loss of appetite, but also the difficulty she was experiencing in buying groceries and cooking for herself and for her husband. As the main caregiver for her husband, her priority was to keep herself and her husband independent in the community and living at home - she did not want to hear about moving either of them to a nursing home. Her daughter is involved but lives far away, and Miss A has been quite depressed with her husband's situation, with her cancer diagnosis and had become completely overwhelmed.

Based on all of these, the geriatrician made recommendations prior to surgery.

- An adjustment and simplification of medication regimen;

- A nutritional consultation;

- A social worker - Korc-Grodzicki mentioned that this was one of the most important recommendations made since she will not be able to take care of herself post surgically if she does not have help for herself and for her husband;

- Treatment of depression;

- Physical therapy for unsteady gait.

We were told that all this is a lot of work. It becomes very difficult for an oncologist or surgeon to do this, as it can take an hour of their time. If the time factor was decreased, then this type of assessment could become more possible for the everyday clinician. One way of doing this is to have screening tools that would basically screen for the need of geriatric assessment. SIOG put a taskforce together to review 17 different screening tests to determine which was more prognostic of an impaired comprehensive geriatric assessment in older cancer patients. Korc-Grodzicki presented the conclusion of this work - "screening tools in older cancer patients should not replace geriatric assessment. However, in a busy clinical practice the use of a screening tool is recommended to identify patients in need of further evaluation by geriatric assessment. No specific tool was recommended or discouraged" [38].

It is better to use a screening tool that takes a few minutes than not to use anything at all.

Korc-Grodzicki presented the model of shared care at her institution, Memorial Sloan Kettering Cancer Center (MSKCC), for patients before or during active cancer treatment. Hers is a geriatric service that is within a cancer center and for the USA this is very unusual. Outpatient and inpatient consultations are provided and everybody that attends the geriatric clinic as a result of being referred for preoperative assessment will have Comprehenisive Geriatric Assessment (CGA). They see about 1200-1300 patients per year for preoperative assessment; every patient will be seen by the geriatrician, a geriatric nurse and a pharmacist - this is the core group. If nutritional therapy, physical therapy or social work is required, then they are called in person as cost restrictions mean they cannot be available within the geriatric service $100 \%$ of the time. The assessment has gone from being a $1-\mathrm{h}$ assessment to being a 15 -min assessment by making the process electronic.

This is termed the 'electronic Rapid Fitness Assessment'. The assessment is made of validated questionnaires that touch on each one of the geriatric domains and the patients are advised that they can complete it on their own, with their caregiver or a family member, or indeed the family member/caregiver can complete it on their own if the patient is unable. If they have an email address they can do this at home, or they can complete it in the clinic waiting area or the examining room while they are waiting for the physician. The electronic Rapid Fitness Assessment touches on all the CGA domains and is basically completely patient centric. Everything is filled out by the patient with the exception of the 'time up and go' - this needs to be done by a nurse and takes at most $30 \mathrm{~s}$, and the 'cognitive assessment' - Mini-Cog is used and that takes $2 \mathrm{~min}$ or so. The information goes to a database that is used for research work, and the information also goes to a report that is printed. The physician will have this report when they see the patient. Sections in the report in red are sections that were not filled out and the physician can then question the patient as to why this section was left blank, and the physician is able to go back and fill in these sections on behalf of the patient. Korc-Grodzicki explained that in their specific patient population, about $40 \%$ 
filled the questionnaire in on their own, about $40 \%$ filled it in with their caregiver and about $20 \%$ of caregivers filled it in alone.

Regarding the geriatric patient population, Korc-Grodzicki summarized that the individual needs an individual cancer treatment that is based not only on the cancer stage, the age of the patient and the cancer therapeutics, but also has to include aspects such as comorbidities, organ function, functional status, cognition, social support, psychological status, polypharmacy, finances and also things like the culture, literacy and the spirituality of the patients. Many patients have different thoughts about cancer and cancer care, and what this means for them and for their families.

Once all this information is gleaned, then it is necessary to act on it. This is termed prehabilitation. Prehabilitation is something that is being looked at in many different ways and is basically a way to make the patient a little bit fitter before they undergo their surgical procedure. Korc-Grodzicki explained that initially, prehabilitation looked at the management of diseases - such as optimizing diabetes or cardiovascular disease, or providing smoking cessation instructions. However, now with functional and geriatric assessments, prehabilitation looks into muscle strength, provision of physical therapy and exercising, nutritional improvements, and emotional and psychological support. When comparing prehabilitation with no prehabilitation, it has been shown that functional recovery is faster with prehabilitation. Improvement in function is seen in both the preoperative and postoperative periods [39].

A study published in 2013 looked at a trimodal prehabilitation program in patients with a mean age of 67 years with a mean program duration of 33 days (this could be a problem depending on the patient; some patients with cancer cannot wait for 33 days to undergo treatment). This study assessed the effects of 30 min walking and breathing exercises three-times a week, a nutritional supplement of up to $1.2 \mathrm{~g} / \mathrm{kg}$ of body weight and anxiety reduction techniques. Results demonstrated that after 4 and 8 weeks, control patients did not reach the presurgical level of physical ability, whereas prehabilitated patients regained the ability to walk farther than their preoperative baseline [40].

Korc-Grodzicki summarized her presentation with the following key points:

- Chronological age in itself is not a reason not to treat;

- Frailty is a predictor of surgical outcomes in the older cancer patient;

- Every older patient needs individualized evaluation to inform any cancer treatment - it has to be patient centric;

- The ideal evaluation is holistic and multidisciplinary;

- Electronic versions were shown to be very helpful and well received;

- Increasing the functional reserve in the preoperative period (prehabilitation) may accelerate the postoperative functional recovery;

- Any evaluation is better than no evaluation at all. Even if there is not much time whatsoever to do an evaluation, just simply asking every patient seen to get off the examining table/get up from the chair, walk 10 feet and sit down again - this 10-20 s of observation could result in a world of information.

Korc-Grodzicki ended her presentation with a quote, "Just as there should be no oncology practice that does not make reference to the TNM staging system, there should be no onco-geriatric practice that does not include frailty assessment" [41].

\section{Oral abstracts: a joint geriatric oncology clinic for the management of elderly women diagnosed} with breast cancer - the Brighton experience

Following Korc-Grodzicki's talk we were presented with a series of four oral abstracts, the first of which was on the topic of 'A joint geriatric oncology clinic for the management of older women diagnosed with breast cancer: the Brighton experience', presented by Fiammetta Ugolini (Brighton and Sussex University Hospital, UK) [42].

By way of background information, Dr Ugolini spoke about how breast cancer incidence increases with age and mentioned that the National Institute of Statistics has shown that the female UK population is made up of over $20 \%$ of women aged 70 years or older. Over $30 \%$ of new diagnoses occur in women over the age of 70 years. As has been mentioned in previous talks during this symposium, older cancer patients are a heterogeneous group and treatment decisions should not be made based on age alone. Dr Ugolini explained that in clinical practice life expectancies are difficult to estimate and should not be confused with chronological age. Indeed, functional and cognitive status may be a better indicator of the suitable therapeutic option. However, it is not easy in day-to-day practice to carry out a comprehensive assessment in a busy diagnostic surgical clinic. 
For all the above reasons, in 2015 the group at Brighton and Sussex University Hospital set up a dedicated clinic in which selected patients could be assessed jointly by a geriatrician and a breast surgeon. The first audit of their clinic activity aimed to look at the impact of joint assessment on the management of breast cancer in the older frail population.

We were presented with various statistics from when the clinic was first set up in June 2015-October 2016. In conclusion, Dr Ugolini stated that assessment in the joint clinic resulted in more patients receiving surgical treatment and issues that had been picked up by the focused geriatric assessment would have very likely not been picked up if patients had not been referred to that clinic.

Oral abstracts: the management of breast cancer in elderly patients - a local audit

The next oral abstract was presented by Taner Shakir (Peterborough Breast Unit, UK) on the topic of, 'The management of breast cancer in elderly patients; a local audit' [43].

Shakir and colleagues decided to evaluate their practice by looking at all the new diagnoses of breast cancer in a 5-year period from the beginning of January 2011 to the end of December 2015 using an age cut off of 75 years.

Shakir presented the results to the symposium, and in conclusion explained that after analyzing these results, it is clear that although surgery was offered to primarily fitter patients and may offer survival benefit, and that there were more complications in the mastectomy group - especially in the over 90s, decisions are multifactorial and all areas spoken about throughout the symposium thus far should certainly be applied.

\section{Oral abstracts: elders with breast cancer tend to delay seeking medical care \& present with a later stage}

The next oral abstract was titled of 'Elders with breast cancer tend to delay seeking medical care and present with later stage' [44] presented by Janice Tsang (Hong Kong Breast Cancer Registry, Hong Kong Breast Cancer Foundation, Hong Kong SAR), speaking on behalf of the Hong Kong Breast Cancer Foundation and the Hong Kong Breast Cancer Registry.

Tsang explained that the fact that breast cancer is the most common female cancer in the UK is also true for Hong Kong and $16-17 \%$ of all breast cancer patients were diagnosed at the age of 70 years or above [45]. It is projected that the population of over 70 s is going to rise and the number of older breast cancer patients will also increase over time. People from Hong Kong are actually on top of the world in terms of longevity and the average life expectation for women is 87 years of age [46].

Tsang presented data from the Hong Kong Breast Cancer Registry which is actually hosted by a nongovernment, nonprofit organization and supported by the Hong Kong Breast Cancer Foundation in the community. Altogether there were 13,265 female patients with breast cancer diagnosed from 2006 to 2015. Among them, 861 of them were aged 70 years or above. About $73 \%$ of patients were treated in the public sector and $27 \%$ were treated in the private sector.

Tsang concluded that this was the first ever comprehensive study of local older breast cancer patients in Hong Kong. The majority of older patients self detected their cancers by chance or as an incidental finding and it takes longer for patients aged 70 years old than for patients of all ages to seek first medical consultation. This suggests an unmet need for increased breast awareness among older patients and their caregivers. Older patients also tend to receive more conservative treatments that have fewer side effects. Comorbidities in older patients could play a role in their treatment choices and further studies are required to look into this point in more detail. As had been mentioned in many of the previous talks, Tsang explained that age may influence breast cancer treatment, but it should not be the sole guiding principle and should not deter the intensity of treatment.

Tsang suggested that patients should be able to assess and choose standard medical treatment if deemed fit. Something unique to the Chinese culture in lots of patients (even some oncologists in China tend not to treat patients aged 70 years and above) is the misconception that they are going to have more side effects. Yet, we know that the older population is a heterogeneous one and should be treated as such. Thus, age should deter the intensity of standard treatment. 


\section{Oral abstracts: potential impact of treatment on the mood of older women of primary breast} cancer

The chair of Session 2, Ruth Parks from the School of Medicine, University of Nottingham, UK, presented the final oral abstract on the topic of, 'Potential impact of treatment on the mood of older women of primary breast cancer' [47].

Parks began by explaining how we had heard already during the symposium thus far that breast cancer in older women differs from their younger counterparts and we know that the treatment for breast cancer is surgery; however, the challenges faced by the older population is very different and can include things like comorbidity and frailty. Furthermore, the biology of breast cancer in older women is different and their tumors are less likely to be as aggressive as those in the younger patient, making the older population more likely to die of other causes than the breast cancer.

These points lead to the consideration of alternative treatments to surgery - for example primary endocrine therapy, and there is growing evidence to suggest that CGA may be able to help in making the decision as to who should be a candidate for surgery and who should receive nonsurgical treatments in unclear scenarios.

Parks and colleagues conducted a prospective two-center pilot study recruiting women over the age of 70 years with newly diagnosed stage I or II breast cancer. CGA was performed at two time points - one within 6 weeks of diagnosis and the second at 6 months post diagnosis. The CGA used a validated, cancer-specific tool, and the group also assessed quality of life with EORTC scoring assessments and conducted semistructured interviews.

After presenting results, Parks concluded that CGA may be helpful in deciding who should have surgery or no surgery treatment in a select cohort. There was an observation of significant difference in mood between surgical and nonsurgical candidates and therefore more work can be done to look into possible mood interventions in these particular patients to improve their quality of life. The study is ongoing, and is being expanded to three centers.

\section{Session 3: patients \& carers}

Following a short break for lunch and poster viewing, delegates reassembled for Session 3. This session began with an interview-style presentation mediated by Heather Stone (Lead Breast Care Nurse Specialist, Royal Derby Hospital) during which three older breast cancer patients were interviewed regarding their experiences since their diagnosis.

The patients were asked questions such as (paraphrased):

- Could you fill us in with what happened when you were diagnosed and what options you were given at the time?

- At what stage were you told what treatments the doctors were going to be recommending?

- One patient's answer to this question was very interesting - the said that they were never advised of any treatment they were going to have, but were told what they could not have - for example, a mastectomy, since they were too old - although the doctors did not put it into so many words, instead saying that it would be too involved and the operation would be about $2 \mathrm{~h}$, and with all the lymph nodes the patient had, it would take another 2-4 h and they would not stand up to it.

- What was the treatment option?

- Can you tell the audience what happens now with your follow-up?

- Were you given the choice of surgery?

- Do you feel that any point you could change your mind and be quite willing to explore other options?

- This patient had previously explained to the audience that they did not want to have surgery although it was offered as one of the proposals at the time.

- When one of the suggested treatments for you was chemotherapy, what was going through your mind when that was proposed?

- How did you get on with your chemotherapy?

- One patient had some news just the other week that they had some local recurrence of the breast cancer and more chemotherapy had been suggested. When asked what their attitude to this was they responded: "Fine. They can start it whenever they like. That's how I feel".

- At any stage of this process, have you felt like a date of birth, a number? Do you feel that you would have been treated any differently if you were $20 / 30$ years younger? 
- To this question, patient responses included, "I think the treatment that I've been given has been 100\%. I could not fault anybody for what I've had and it's all been done so quick". And, "it's diagnosed and you get on with the job and that's it", and "I have to agree. It's been excellent. The treatment has been fantastic".

- Is there anything you would change apart from your diagnosis?

At this point the floor was open for questions.

One delegate asked, "What sort of information did you have when you were being given your options? Was it just a talk with the doctor, or was it with the nurse or did you have other information and how useful did you find it?"

Patient answers included (paraphrased), "I didn't read the contents of the envelope I was given at all. And I didn't like at that stage what I was told because it was all gloom and doom. I didn't want to know about it. But subsequently I'm very happy about it", "I was given more information on chemotherapy and radiotherapy - you could fill a book case. It was useful. You read it and put it away".

Another question posed by an audience member to a specific patient was, "Do you feel that because you were under investigations for other things that were going on, do you feel that influenced your decision about the diagnosis and the treatment thereafter for the breast cancer?"

This patient explained (paraphrased), "No, I don't think so. It's just something I've had in my mind for years. I have had surgery but not for cancer. Operations - I just go ahead with it as you do, but with cancer I've always had this feeling that once you cut it spreads and I am firmly convinced of it. And so I would never accept surgery. I don't know what goes on afterwards and I don't know how I will finish up, but I don't want surgery".

The patient had spoken to her doctor about this and they were well aware of how she felt.

Another important question was regarding the cost of care.

One patient answered that altogether the cost of a taxi to the hospital and back was about $£ 13$ and had to make about one trip every 3 weeks when on chemotherapy and had to go in everyday when undergoing radiation therapy, and the cost therefore mounts up.

Other cost related issues that came up were the cost of parking and time taken off work by relatives/carers.

One of the final questions asked by the audience was "At the time that you had the diagnosis of breast cancer and you were going to look forward into the future, what things were you most worried about and what were the things that you most wanted to preserve best?"

The answers from the patients to this question were very inspiring (paraphrased).

One patient said, "Nothing really. At my age you don't worry. I was so happy eventually with the care that was taken of me I cancelled my private insurance."

Another answered, "Well, I've always though there's no use worrying about the past-it's gone. There's no use bothering about what's going to happen in the future, you don't know what's going to happen or what's round the corner. Live for today, Just that's it."

The final patient said, "Again, I'm quite happy with the treatment I've had. We couldn't have been in better care. You just live life for today and that's what you've got to do. When I was diagnosed at first I was supposed to have had abscesses. I was treated for 5 weeks for abscesses and it comes out at the end it wasn't. It was cancer. So you live for today. Do as you've always done. So long as I can get to the shops every morning I don't care a dime."

\section{The role of the geriatric oncology nurse}

After the patient interview session, Cindy Kenis (Geriatric Oncology Nurse, University Hospitals Leuven, Belgium) presented on 'The role of the geriatric oncology nurse'.

Dr Kenis began by saying that 'older persons' are a heterogeneous population. As we grow older we become more and more different from each other. She also spoke of how 'cancer and aging' is the challenge of the century. As many other speakers had already mentioned, chronological age is not the same as the biological age. When presented with three people, each 30 years of age, one could probably guess that they are all functioning in a similar way. Conversely, when three women of 85 years of age are presented - they would most probably be very different. In the clinic when you see an older woman with a new diagnosis of breast cancer, there are so many questions you can ask yourself:

- What does this lady do?

- Where does she come from?

- How does she function in daily living? 
- How good is her memory?

- What things does she do in her free time or when she is at home?

It can be difficult to make specific treatment decisions for older patients with cancer. In daily practice many questions can arise, such as:

- Do further diagnostic examinations have therapeutic implications in this patient? For example, what implications does a diagnosis of dementia or other geriatric comorbidity have for an 85 -year-old woman with a new diagnosis of breast cancer?

- Is the patient 'too old' or 'not too old' for therapy?

- What is her life expectancy? A woman of 85 years of age and a woman of 95 years of age, both with a new diagnosis of breast cancer will have different and specific life expectancies.

- Will he or she tolerate therapy? Patients at a breast cancer clinic could be faced with hormonal therapy, chemotherapy, radiation therapy and now, many new therapies are being developed such as targeted therapies and biological agents - all therapies with potential side effects as you could have with chemotherapy.

- Has the patient enough support to maintain therapy? For example, patients with breast cancer may have to come to the clinic for 6 weeks for radiation therapy. Can they come every day? Have they got someone to come with them or can they come alone?

CGA is therefore the key aspect in the care of older patients and has already been for some decades, the cornerstone of geriatric medicine. Dr Kenis explained that CGA goes about, first, identifying patients in need of geriatric assessment and, second, evaluating those patients with geriatric screening and geriatric assessment based on validated instruments. Based on the results of the assessment, third, recommendations need to be developed and these are related to the interventions that can be planned in the care plan. The recommendations then need to be, fourth, implemented and, fifth, provision has to be made for follow-up.

The main goal is to see if a patient is fit or frail. It is not too difficult to recognize a very fit and a very frail patient, but we find that the majority of older patients that we see are a group in between these two extremes.

Kenis went on to talk about how this is currently being implemented in general in the world [48]. First, there is the geriatric assessment ward/geriatric unit. Most hospitals have a unit where all the patients are hospitalized and there are two different units here - the geriatric evaluation and management unit, and the acute care for elders unit. Second, there is the geriatric consultation team - they are very specific for patients who are not staying in a geriatric unit and a nurse or another healthcare professional brings geriatric expertise based on CGA. Third, there is the new concept of co-management. Co-management units have a very specific focus on for example, orthogeriatric (e.g., hip fracture).

Specifically to geriatric oncology, we were told that the following three things can be observed:

- There are countries with developed geriatric oncology units.

- There is the same concept as the geriatric consultation team - so a healthcare professional brings the geriatric expertise to the oncologic in- and outpatients of the hospital.

- A model like in the USA where there are comprehensive cancer centers - where geriatric expertise is not available nearby, so they receive educational material about how to combine geriatric expertise and integrate this in care plans for oncology patients.

There are many ways to implement everything related to CGA in daily practice and they all have advantages and disadvantages [48]. Kenis explained that the choice is very much related to very country-specific reasons and structures, and there is currently no information on which model is the best. Interaction with multidisciplinary geriatric teams is highly recommended and in all models the role of the nurse and their educational background is different.

The model of geriatric oncology nursing basically combines all of the concepts of oncology, geriatrics and nursing but extends beyond traditional management of the illness and focuses on all other aspects that are relevant for patients [49].

Kenis then went through the five-step approach to CGA step by step. 
- Screening: had already been mentioned quite a lot during the symposium. It is performed with a screening tool and there are many different screening tools available such as G8, Flemish version of the Triage Risk Screening Tool, Groninger Frailty Index among others. None of these tools should replace a full geriatric assessment, but this does not mean that the screening tools do not have any meaning. They are important and are quick and fairly simple to use. With their combination of questions, one can get a small overview of some specific areas. A person with a normal result on a screening tool often performs better with regards to overall survival. They do not have high risk for functional decline, falls and so on. If the result with the screening tool is abnormal then a geriatric assessment will need to be performed to explore where the specific problem areas are.

- Geriatric assessment: this explores all the domains relevant as has already been touched on during the symposium, namely, social support, functional status, fatigue, comorbidity, cognition, mental health, nutritional status and geriatric syndromes, such as falls and polypharmacy. Various tools are available to investigate these domains and it has not been proven regarding which tool is best. The choice of which instrument to use could rely on the local preference, aim of the tool and/or the resources present.

- Geriatric recommendations: established based on the results of the geriatric assessment. The geriatric oncology nurse will be available to communicate and to comment to optimize the care/treatment plan.

- Geriatric intervention: Dr Kenis posed the question that if you discover following the geriatric assessment that the patient has a nutritional problem, does that mean it should be solved before treatment starts? The answer is 'no'. When the existence of a nutritional problem is known, it can then be integrated in to the care plan of the patient to make sure that something is done to target the nutritional problem from the moment the treatment of that patient starts. Interventions can sometimes be very difficult. The results of the assessment and recommendations could mean that other healthcare professionals need to get involved - for example, the fall clinic, the memory clinic among others. For some hospitals in Belgium, every patient that comes in with a new cancer diagnosis will always see a psychologist, social worker and a dietician. Is this necessary? Do patients need to see all those healthcare professionals systematically in each case? Some answer yes, some answer no.

\section{- Follow-up}

Why does CGA need to be performed and why is it being integrated in to daily oncology practice? Kenis explained that this assessment detects unidentified geriatric problems. Geriatric assessment also has a prognostic and predictive value for the patient. The two main issues that are prognostic and predictive are nutritional and functional status based on (inter)national studies. We were told that patients who have a bad nutritional status, very often perform much worse on overall survival and also on the experience of treatment-related toxicity and complications.

Geriatric assessment can also influence treatment decisions, not just which therapy to give, but also whether to increase or decrease therapy intensity. The decision can sometimes be that we need to start palliative care for example. Finally, geriatric assessment can also allow more targeted interventions which can improve quality of life and overall survival. Targeted interventions can never be based on the geriatric screening only. Screening will only give information about who has more of a chance for overall survival or an increased risk for functional decline, but interventions can only be based on the results of the assessment.

In conclusion Kenis spoke of how geriatric oncology nursing is very relevant. Many nurses working in the field of geriatric oncology have an extremely good oncology background but still need additional education about geriatrics and other things also. An ideal model of care in geriatric oncology nursing needs to integrate the concepts of oncology, geriatrics and nursing care. The role of the 'nurse' whether specifically a geriatric oncology nurse or not, is crucial in all existing models that are currently described in the literature. Education of oncology nurses is necessary - in the concepts of geriatrics and they also need exposure to the use of geriatric assessment and screening tools.

In general, Kenis said that healthcare professionals need to be watchful for age-related aspects as they are important and relevant. CGA is very useful as was mentioned previously in Kenis' presentation. Overall, geriatric recommendations and interventions increase CGA effectiveness with the final goal of improving quality of care for older patients with (breast) cancer.

Session 3 ended with a short discussion and was then adjourned for a coffee break and poster viewing. 


\section{Session 4: challenging areas}

\section{Selecting for breast reconstruction}

The first talk for the final session of the symposium was given by Graeme Perks, Consultant Plastic and Reconstructive Surgeon, Nottingham University Hospitals; Immediate Past President, British Plastic Reconstructive and Aesthetic Surgeons (BAPRAS) on the topic of, 'Selecting for breast reconstruction'.

Mr Perks began by saying that when facing a patient in a consultation, it is important to ask 'what can I do to help you have more fun in life?' He talked of how he has tried, every time he meets a patient, to ask them what their interests and hobbies are, who they have to share a life with, what their career has been or what the highlight of their career has been. Everybody has a story and it is good to ask about it.

People often think that breast reconstruction is simply about implants - however, Mr Perks explained that he thought that the key to breast reconstruction is looking beyond the breast implant.

There have been many issues associated with implants over the years; silicone gel implant inquiries, Trilucent ${ }^{\mathrm{TM}}$ (Lipomatrix Inc., Neuchâtel, Switzerland) breast Implant inquiries (marketed under the Freudian description that they were filled with soya oil and therefore safe enough to eat) and titanium-coated breast implants that came from Germany and then it was discovered that they were being put into patients and they did not have a Conformité Européenne (CE) mark! The spinoff from that was titanium mesh which some of the surgeons would use as a part of breast implant reconstruction that has become widely accepted as an adjunct to implant-based reconstruction.

Perks explained how implants have many problems associated with them, the biggest problem being scar tissue that collects around the implant. The body does not reject the foreign object it recognizes, but it can tightly compress the foreign object. Another problem with breast implants is that they do not always stay where you put them.

When choosing for breast reconstruction, one can consider the three-question algorithm:

- What is missing?

- What is required?

- What is available?

It is also important to remember that there is a patient involved in all this - it is about an individual.

One thing a reconstructive surgeon aspires to is to try and put the patient back where possible to as close as they were before they had the problem - this is not that easy to achieve.

Perks outlined the various considerations a breast reconstructive surgeon has to think about for each patient namely, the tumor size, the breast volume, the relative proportions of the tumor and the breast, what is going on in the opposite breast (for older patients it is much more likely that the opposite breast will need something to be done to them, because they are likely to be droopy), you need to look at the patient as a whole and then you need to wonder about their musculoskeletal comorbidity - have they got back pain (if the tummy is operated on, the latissimus dorsi - the core muscles that affect the patient's skeletal support, is altered) or shoulder pain problems? Have they got hernias? One rule of thumb Perks highlighted to delegates that he used was that, if a patient's tummy sticks out further than their breasts, he is very unlikely to use tissue from their abdomen - usually because the muscle layer is too weak and they have got too much fat.

It is important to keep questioning methods and decisions and checking whether that is the right operation for the right patient. Perks explained that some years ago, he, along with his colleagues, looked at morbidity - where they took the flap from the tummy using the rectus-abdominis muscle entirely as opposed to taking just the blood supply (that is the main difference of a TRAM flap [having to take all the muscle] and the DIEP flap [where you just take the blood vessels]), and they showed there was no difference [50].

When beginning a reconstruction, it is the patient and their expectations/hobbies among others that should be taken into consideration. Perks explained that he starts out asking about the person first, and then ask them what they understand about the disease because it was necessary to build up a picture about the patient before worrying about why they have come.

It is also important to know about vascular supply, what is going to happen with the oncologist (chemotherapy/radiation therapy), what the surgeons are planning to do among others. In other words - it is necessary to know about your team.

The question is, "will the process be breast reconstruction or mastectomy?" If the breast can be conserved, that is definitely better, whether primary or delayed reconstruction is carried out. The patient may well say, "I can not 
get my head around how you reconstruct, so let me deal with the mastectomy first and then I'll come and talk about the reconstruction". Perks explained that some of the happiest patients have been patients who have decided not to have reconstruction. It is very important to make sure the patient understands what the cost of reconstruction will be to their bodies.

Perks presented the 'KISS' principle - 'Keep It Simple, Stupid!' and explained that as breast reconstructive surgeons, they try and apply this wherever possible, especially when it comes to training the trainees not to get ahead of themselves and think that they are going to have complicated, exciting surgeries - but keeping it safe and simple.

Another aspect that needs to be considered is the many costs to the patient - whether it be a physical cost, psychological cost (not all hospitals have resident psychologists), relationship costs (some patients may say, "now, my husband won't go near me"; you can do the best surgery in the world, but this may affect the relationship), employment costs and financial costs.

Perks once again stressed that the whole procedure needs to be about the patient and her expectations. The patient and her partner needs to be educated in order for them to make an informed decision and understand risks. They need to be supported by the surgeon and a specialist nurse. Patient and partner education should also explore reconstructive options and provide them with an understanding of the range of additional procedures that may be required.

Vascular supply can be affected by smoking, cardio-respiratory problems (Perks included diabetes here), obesity (fat is a parasite and does not add any benefit to the wound healing), problems with the skin envelope, ptosis, where you put your incision and radiation therapy (if you have wide-lobe excision - it causes endarteritis obliterans).

Blood supply is important. The surgeon will be faced with problems with the blood supply and wound healing unless they bear in mind that every patient is an individual. Technology is now available that allows a surgeon to check out the blood supply to the abdomen and when doing flap reconstruction allows for the biggest vessel to be picked out [51]. Lord Moynihan stated that "the smoother and gentler the act of surgery, the more exquisite the wound healing." As a surgeon it is necessary to be really gentle and treat the tissue with respect.

An oncologist needs to perform chemotherapy and radiation therapy prior to surgery. Questions are being asked about whether you can give - Group 1: immediate reconstruction and radiation therapy; Group 2: delayed reconstruction after radiation therapy; Group 3: immediate reconstruction without radiation therapy. This comparison is ongoing in order to see what can be done to improve outcomes. Once the breast is irradiated, the blood supply has been damaged so it is not a great idea to operate on irradiated breast tissue.

Perks explained that breast reconstruction includes lumpectomy, wide local excision and implants - a cut is being made and then being sewed up again. Then there is breast conserving therapy - perform wide local excision and reshape the breast, or local flaps can be used. Perks highlighted mammoplasty as one of the greatest advances in his opinion, allowing breast conserving therapy to be carried out in cases where there might be poor cosmesis. Here, you can get good access to the tumors and avoid a mastectomy and total breast reconstruction in many cases. Mammoplasty is really good for other things also - particularly in women who have got droopy breasts or large breasts - effectively, they can be given a breast reduction to give good sensation. It could probably help with problems of radiation therapy in large breasts also.

The bottom line is to turn up to surgery fully prepared to avoid any problems in procedures.

Lipotransfer is gaining popularity. Fat is aspirated and then this can be injected into the breast, but there is a lot that we do not understand here yet although work has been done in this area [52,53]. In order for fat grafting for breast reconstruction to be carried out, you have to have a certain amount of fat available. There have been concerns about it possibly promoting cancer progression, so fat grafting is not without its problems.

Perks concluded by explaining that we need to remember it is about the patient and the patient's partner. Work has to be done along with the oncologist, the breast cancer surgeon and the breast care nurse to understand from the patient what would work best for them and then really listen to what they are saying in order to get the best outcomes.

HER2 + breast cancer in elderly

The final talk in Session 4 was on the topic of 'HER2+ breast cancer in elderly' and was presented by Etienne Brain (Medical Oncologist, Institut Cure, Paris, France). 
Dr Brain began by explaining that HER2+ disease comprises a small segment of the breast cancer patients, and it best exemplifies the great strides in treatment personalization that we have seen during the past almost 20 years. Why is HER2+ disease important?

Dr Brain went right back to the first introduction of the semihumanized monoclonal antibody by Roche trastuzumab. This was progressively introduced and provided outstanding results in combination with chemotherapy. An editorial paper published after the introduction of trastuzumab in the adjuvant setting following ASCO 2005, stated that "in our opinion, there have been only two walk-off home run oncology products or strategies in the last 20 years - ones that provided relative reductions exceeding $50 \%$ compared with the best current care imatinib and trastuzumab" [54]. There is a high cost associated with this however, and all personalized medicines consistently increase the cost in our healthcare system. Looking at the data in the adjuvant setting, what trastuzumab has achieved in terms of results on progression-free survival is quite remarkable as it is quite consistent across large trials with a reduction of the risk of relapse and the risk of death.

In trials, one can see that the numbers of older patients included in the trials were a small segment - usually between 10 and $15 \% ; 16 \%$ in the HERA trail, and this does not match up to the reality of what we treat. Just because the HER2 segment is small in the older population it does not mean that we should have such a low representation in trials. Dr Brain highlighted the fact that in older patients there is cardiac toxicity (congestive heart failure) that is observed sometimes with chemotherapy and in particular anthracyclines - and there is certainly a higher risk with trastuzumab. One combined analysis of various trials demonstrated that congestive heart failure cases increase with the use of trastuzumab in the adjuvant setting and age is a factor to increasing that risk [55].

Results from analysis of the Surveillance, Epidemiology and End Results (SEER) program database for patients 66 years of age or older, show that during the period of 2005-2009, the use of trastuzumab concentrates to the below 75 years age group. It is not used very much in really older patients and the use of trastuzumab decreased with comorbidities associated with older age [56]. There is also a risk that the officially approved 1-year treatment schedule is not completed. Very often, these patients do not complete their whole 12 months of antibody treatment and we see up to $10 \%$ of hospitalizations due to cardiac events in the year following the completion of treatment. This does not really match the picture obtained from clinical trial results as they do not include enough patients to get strong conclusions.

Dr Brain presented results of a paper published in the Journal of Clinical Oncology, showing the receipt of trastuzumab among women with HER2+ breast cancer by stage and race [57]. He also explained that when trastuzumab is used in combination with chemotherapy there are many variations seen across the world. In the USA very often, TCH (docetaxel, carboplatin and trastuzumab) is used - makes up around $40 \%$ of cases, trastuzumab with taxane accounts for $21 \%$ of cases, trastuzumab with single-agent chemotherapy accounts for $14 \%$ of cases, ACTH (doxorubicin, cyclophosphamide, paclitaxel and trastuzumab) accounts for about $9 \%$ of cases, and trastuzumab with other agents for about $8 \%$ of cases [57]. TCH was clearly quite important here, however, Dr Brain explained that TCH use in his practice, as well as in France/Europe is quite limited.

Dr Brain also showed that TCH decreases the amount of cardiac toxicities in the adjuvant setting and there are alternatives to the standard chemotherapy usually used in combination with trastuzumab, and also an option for avoiding anthracyclines with a potential benefit for the older patients.

There has been a long history of different antiHER2 compounds. Lapatinib was developed as a tyrosine kinase inhibitor (TKI) to act on the HER2 internally in tumor cells. There was a long 'fight' between lapatinib - the light TKI and trastuzumab - the heavy antibody. The TKI actually has many more issues of pharmacological interactions with co-medications which are increased in the older populations.

Dr Brain also presented a comparison between pertuzumab and T-DM1 (Kadcyla).

One of the most achieved results was the CLEOPATRA trial that looked at pertuzumab + trastuzumab + docetaxel versus placebo + trastuzumab + docetaxel, in a metastatic first line setting [58]. Using two antibodies was extremely active showing a 6-month benefit in progression-free survival leading to a new standard of care. A specific analysis according to age shows that for a predefined threshold of 65 or 75 , it appears that two antibodies versus one do the same irrespective of age [59]. The safety profile is different however, and there is more diarrhea, asthenia, fatigue, decreased appetite, vomiting and dysgeusia (any grade) in older patients [60]. Dr Brain highlighted the importance of taking the fact that in this large trial of 800 patients, only 19 were over 75 years of age into account.

Another possibility is using dual antibodies without chemotherapy - thus avoiding the burden of side effects that chemotherapy brings [61]. This kind of challenge is quite important in terms of development as older patients 
are more sensitive to chemotherapy. If this burden of side effects could be skipped and only targeted therapy be used it could potentially be great. Dr Brain stated that as a concept this is quite attractive; however, it needs to be investigated and demonstrated.

The concept of dual blockade is not clear. The ALTTO study tried to look at the disease-free survival (DFS) using lapatinib plus trastuzumab in an adjuvant setting and failed - there was no difference across the different curves between dual or single blockade. A very important trial - the MARIANNE study was published last year and compared dual blockade versus not and this also failed to see an impact of the dual blockade [62].

The most recent results are with regards to the Phase III APHINITY study. This is a randomized trial in an adjuvant setting - looking at the use of one or two antibodies in adjuvant chemotherapy - trastuzumab plus pertuzumab versus trastuzumab placebo for 1 year. The press release reports that it met primary criteria on DFS and this could therefore change the landscape of HER2+ disease [63].

EORTC is looking into this as well - older patients were randomized to a strategy skipping chemotherapy and using both antibodies versus a standard combination of dual antibodies plus metronomic chemotherapy. This trial has completed accrual. Another Swiss trial is going to compare exactly the same strategy - trying to skip chemotherapy and benefit the most from the dual blockade in the adult population with some older patients. Results of both these trials can be combined to hopefully obtain some conclusive data.

Dr Brain explained that there is a task force at SIOG, which has reviewed all the literature on this specific hot topic working for almost 2 years on the review (publication pending). In this they have reached a consensus with the general message being, that these kinds of treatments which are part of innovation and important for the older population can be used but we cannot usually extrapolate what has been shown in trials as they have been done only on a fixed segment of the population. He also raised the issue of cost. A hot debate in ASCO 2016 reminded the audience that the cost-effectiveness of the HER2+ anti-HER2 treatment is only demonstrated for adjuvant treatment in adjuvant settings for 1 year, and in metastatic settings for the single blockade. As soon as you integrate the dual blockade in medicine, things change a lot and it depends on the price according to specific countries.

It is important to introduce more older patients in future trials, as well as introducing more of a focus on quality of life - in Dr Brain's opinion - this is very, very important as quality of life clearly arbitrates the choices that we make.

\section{Conclusion \& future perspectives}

Following a discussion of the two talks in Session 4, David Morgan, Consultant Clinical Oncologist (retired), Nottingham University Hospitals, and Symposium co-chair, presented the main concepts that in his opinion had come through during the symposium talks.

First, he explained that a number of presenters have pointed out that the question of how to manage older patients with primary breast cancer is a big problem and the problem is getting bigger. There are going to be more old patients - almost coming to an epidemic of older breast cancer. These discussions, as presented in the symposium are very, very important for future practice in oncology.

Additionally, the balance of treatment has been touched on so many times. Essentially one is walking on a tightrope of overtreatment (harm) and undertreatment (not doing enough to combat the cancer). Dr Morgan thought that what had been emerging during the symposium talks and what has become clearer over time is that the question was, "Well, there is an issue of deciding how a patient is going to be able to tolerate the treatment and we need to look into this". In the past the question had been "Can we do this?"; now, the question is "How do we do it?".

There have been a number of presentations on the different tools that are available and have been shown to be useful, and there has been a big progress in the field of managing older patients, and weighing up benefits and harms of treatments. Much of this has come through the involvement of geriatricians in the process of deciding treatment for older patients. Morgan explained that this is something we should strive to do more and more in clinics - to involve geriatricians who know about the tools that are available, how to use them and how they should be applied.

The concept of frailty was also raised during the symposium and its significance has been much more widely appreciated in recent years. It is a good way of describing how you are going to assess a patient in terms of her ability to tolerate treatments that are sometimes aggressive.

Morgan highlighted that all speakers and Graeme Perks, in particular, had raised the importance of establishing how the patient feels about treatment, and Morgan stated that he did not think we could emphasize too strongly how important this is. Hearing from the patients during the patient interview segment of the symposium demonstrated 
how they liked to be involved. The patients need to be asked how they feel and physicians need to ensure that they are going along with what they and their carers think is right.

One last important issue that Morgan highlighted as coming through the conference was that biology of the tumors is different in older patients. The very first talk in the very first symposium many years previously was on the difference in the biology of cancer in the older patients. What is clear is that while many cancers are aggressive and kill patients (and therefore they should not be undertreated), in older patients, in many cases the cancer is going to 'outlive' the patients and these patients will die of something other than their breast cancer. It is therefore important not to harm their quality of life by applying treatment that is unnecessary, and this needs to be considered very carefully when making treatment decisions.

Dr Morgan ended by thanking all those who attended the conference and also all the speakers.

\section{Financial \& competing interests disclosure}

The authors have no relevant affiliations or financial involvement with any organization or entity with a financial interest in or financial conflict with the subject matter or materials discussed in the manuscript. This includes employment, consultancies, honoraria, stock ownership or options, expert testimony, grants or patents received or pending, or royalties.

No writing assistance was utilized in the production of this manuscript.

\section{References}

1 4th symposium on primary breast cancer in older women. Future Oncol. 13(8s), 1-18 (2017).

2 University of Nottingham. 4th symposium on primary breast cancer in older women. www.nottingham.ac.uk/conference/fac-mhs/medicine/symposia-on-primary-breast-cancer-in-older-women/resources.aspx

3 SIOG. International Society of Geriatric Oncology. www.siog.org

4 de Kruijf EM, Bastiaannet E, Rubertá F et al. Comparison of frequencies and prognostic effect of molecular subtypes between young and elderly breast cancer patients. Mol. Oncol. 8(5), 1014-1025 (2014).

5 Jenkins EO, Deal AM, Anders CK et al. Age-specific changes in intrinsic breast cancer subtypes: a focus on older women. Oncologist 19(10), 1076-1083 (2014).

6 Schonberg MA, Marcantonio ER, Li D, Silliman RA, Ngo L, McCarthy EP. Breast cancer among the oldest old: tumor characteristics, treatment choices, and survival. J. Clin. Oncol. 28(12), 2038-2045 (2010).

7 van de Water W, Markopoulos C, van de Velde CJ et al. Association between age at diagnosis and disease-specific mortality among postmenopausal women with hormone receptor-positive breast cancer. JAMA 307(6), 590-597 (2012).

8 Muss HB, Woolf S, Berry D et al. Adjuvant chemotherapy in older and younger women with lymph node-positive breast cancer. JAMA 293(9), 1073-1081 (2005).

9 Giordano SH, Duan Z, Kuo YF, Hortobagyi GN, Goodwin JS. Use and outcomes of adjuvant chemotherapy in older women with breast cancer. J. Clin. Oncol. 24(18), 2750-2756 (2006).

10 Elkin EB, Hurria A, Mitra N, Schrag D, Panageas KS. Adjuvant chemotherapy and survival in older women with hormone receptor-negative breast cancer: assessing outcome in a population-based, observational cohort. J. Clin. Oncol. 24(18), 2757-2764 (2006).

11 de Glas NA, van de Water W, Engelhardt EG et al. Validity of adjuvant! Online program in older patients with breast cancer: a population-based study. Lancet Oncol. 15(7), 722-729 (2014).

12 de Glas NA, Bastiaannet E, Engels CC et al. Validity of the online PREDICT tool in older patients with breast cancer: a population-based study. Br. J. Cancer 114(4), 395-400 (2016).

13 Prat A, Perou CM. Deconstructing the molecular portraits of breast cancer. Mol. Oncol. 5(1), 5-23 (2011).

14 Cardoso F, van't Veer LJ, Bogaerts J et al. 70-gene signature as an aid to treatment decisions in early-stage breast cancer. N. Engl. J. Med. 375(8), 717-729 (2016).

15 Shak S, Miller DP, Howlader N et al. Outcome disparities by age and 21-gene recurrence score result in hormone receptor-positive (HR+) breast cancer. Ann. Oncol. 27(6), 43-67 (2016).

16 ClinicalTrials.gov. 'Adjuvant systemic treatment for (ER)-positive HER2-negative breast carcinoma in women over 70 according to genomic grade (GG): chemotherapy + endocrine treatment versus endocrine treatment (ASTER 70s)'. https://clinicaltrials.gov/ct2/show/NCT01564056

17 Bartlett JM, Bayani J, Marshall A et al. Comparing breast cancer multiparameter tests in the OPTIMA prelim trial: no test is more equal than the others. J. Natl Cancer Inst. 108(9), pii:djw050 (2016).

18 Oeppen J, Vaupel JW. Demography. Broken limits to life expectancy. Science 296(5570), 1029-1031 (2002). 
19 Allemani C, Minicozzi P, Berrino F et al. Predictions of survival up to 10 years after diagnosis for European women with breast cancer in 2000-2002. Int. J. Cancer 32(10), 2404-2412 (2013).

20 Early Breast Cancer Trialists' Collaborative Group (EBCTCG), Darby S, McGale P, Correa C et al. Effect of radiotherapy after breast-conserving surgery on 10-year recurrence and 15-year breast cancer death: meta-analysis of individual patient data for 10,801 women in 17 randomised trials. Lancet 378(9804), 1707-1716 (2011).

21 Blamey RW, Bates T, Chetty U et al. Radiotherapy or tamoxifen after conserving surgery for breast cancers of excellent prognosis: British Association of Surgical Oncology (BASO) II trial. Eur. J. Cancer 49(10), 2294-2302 (2013).

22 Technical Basis of Radiation Therapy. Practical Clinical Applications (5th Edition). Levitt SH, Purdy JA, Perez CA, Poortmans P (Eds). Springer, Berlin Heidelberg, Germany (2012).

23 Poortmans PMP, Arenas M, Livi L. Over-irradiation. Breast 31, 295-302 (2017).

24 ClinicalTrials.gov. 'Radiation dose intensity study in breast cancer in young women'. https://clinicaltrials.gov/show/NCT00212121?link_type=CLINTRIALGOV\&access_num=NCT00212121

25 van Maaren MC, de Munck L, de Bock GH et al. 10 year survival after breast-conserving surgery plus radiotherapy compared with mastectomy in early breast cancer in The Netherlands: a population-based study. Lancet Oncol. 7(8), 1158-1170 (2016).

26 Polgár C, Van Limbergen E, Pötter R et al. GEC-ESTRO Breast Cancer Working Group. Patient selection for accelerated partial-breast irradiation (APBI) after breast-conserving surgery: recommendations of the Groupe Européen de Curiethérapie-European Society for Therapeutic Radiology and Oncology (GEC-ESTRO) breast cancer working group based on clinical evidence (2009). Radiother Oncol. 94(3), 264-273 (2010).

27 Smith BD, Arthur DW, Buchholz TA et al. Accelerated partial breast irradiation consensus statement from the American Society for Radiation Oncology (ASTRO). Int. J. Radiat. Oncol. Biol. Phys. 74(4), 987-1001 (2009).

28 Iyer VR, Eisen MB, Ross DT et al. The transcriptional program in the response of human fibroblasts to serum. Science 283(5398), 83-87 (1999).

29 Chang HY, Sneddon JB, Alizadeh AA et al. Gene expression signature of fibroblast serum response predicts human cancer progression: similarities between tumors and wounds. PLoS Biol. 2(2), E7 (2004).

30 Nuyten DS, Kreike B, Hart AA et al. Predicting a local recurrence after breast-conserving therapy by gene expression profiling. Breast Cancer Res. 8(5), R62 (2006).

31 Makary MA, Segev DL, Pronovost PJ et al. Frailty as a predictor of surgical outcomes in older patients. J. Am. Coll. Surg. 210(6), 901-908 (2010).

32 Balducci L, Extermann M. Management of cancer in the older person: a practical approach. Oncologist 5(3), 224-237 (2000).

33 Bouchardy C, Rapiti E, Fioretta G et al. Undertreatment strongly decreases prognosis of breast cancer in elderly women. J. Clin. Oncol. 21(19), 3580-3587 (2003).

34 Ugolini G, Ghignone F, Zattoni D, Veronese G, Montroni I. Personalized surgical management of colorectal cancer in elderly population. World J. Gastroenterol. 20(14), 3762-3777 (2014).

35 NCCN, Senior Adult Oncology, Version 2.2016.

36 Robinson TN, Eiseman B, Wallace JI et al. Redefining geriatric preoperative assessment using frailty, disability and co-morbidity. Ann. Surg. 250(3), 449-455 (2009).

37 Korc-Grodzicki B, Sun SW, Zhou Q et al. Geriatric assessment as a predictor of delirium and other outcomes in elderly patients with cancer. Ann. Surg. 261(6), 1085-1090 (2015).

38 Decoster L, Van Puyvelde K, Mohile S et al. Screening tools for multidimensional health problems warranting a geriatric assessment in older cancer patients: an update on SIOG recommendations. Ann. Oncol. 26(2), 288-300 (2015).

39 Carli F, Gillis C, Scheede-Bergdahl C. Promoting a culture of prehabilitation for the surgical cancer patient. Acta Oncol. 56(2), 128-133 (2017).

$40 \mathrm{Li} \mathrm{C}$, Carli F, Lee L et al. Impact of a trimodal prehabilitation program on functional recovery after colorectal cancer surgery: a pilot study. Surg. Endosc. 27(4), 1072-1082 (2013).

41 Audisio RA, van Leeuwen B. When reporting on older patients with cancer, frailty information is needed. Ann. Surg. Oncol. 18(1), 4-5 (2011).

42 Ugolini F, Wright J, Reed M. A joint geriatric oncology clinic for the management of elderly women diagnosed with breast cancer: the Brighton experience. Future Oncol. 13, 8s, 2017. 4th symposium on primary breast cancer in older women, March 2017, Nottingham, UK.

43 Shakir T, Goh S. The management of breast cancer in elderly patients: a local audit. Future Oncol. 13, 8s, 2017. 4th symposium on primary breast cancer in older women, March 2017, Nottingham, UK.

44 Tsang J, Cheung P, Lee $\mathrm{H}$ et al. Elders with breast cancer tend to delay seeking medical care and present with a later stage. Future Oncol. 13, 8s, 2017. 4th symposium on primary breast cancer in older women, March 2017, Nottingham, UK.

45 Hong Kong Cancer Registry. Hospital Authority (2012). www3.ha.org.hk/cancereg 
South China morning post (28 July 2016).

www.scmp.com/news/hong-kong/health-environment/article/1995947/hong-kong-women-and-men-enjoy-worlds-longest-life

47 Parks RM, Cox K, Howard P, Cheung KL. Potential impact of treatment on the mood of older women with primary breast cancer. Future Oncol. 13, 8s, 2017. 4th symposium on primary breast cancer in older women, March 2017, Nottingham, UK.

48 Wildiers $\mathrm{H}$, Heeren P, Puts $\mathrm{M}$ et al. International Society of Geriatric Oncology consensus on geriatric assessment in older patients with cancer. J. Clin. Oncol. 32(24), 2595-2603 (2014).

49 Burhenn PS, Perrin S, McCarthy AL. Models of care in geriatric oncology nursing. Semin. Oncol. Nurs. 32(1), 24-32 (2016).

50 Schaverien MV, Perks AG, McCulley SJ. Comparison of outcomes and donor-site morbidity in unilateral free TRAM versus DIEP flap breast reconstruction. J. Plast. Reconstr. Aesthet. Surg. 60(11), 1219-1224 (2007).

51 Schaverien MV, Ludman CN, Neil-Dwyer J et al. Contrast-enhanced magnetic resonance angiography for preoperative imaging in DIEP flap breast reconstruction. Plast. Reconstr. Surg. 128(1), 56-62 (2011).

52 Sinna R, Delay E, Garson S, Delaporte T, Toussoun G. Breast fat grafting (lipomodelling) after extended latissimus dorsi flap breast reconstruction: a preliminary report of 200 consecutive cases. J. Plast. Reconstr. Aesthet. Surg. 63(11), 1769-1777 (2010).

53 Martin-Padura I, Gregato G, Marighetti P et al. The white adipose tissue used in lipotransfer procedures is a rich reservoir of CD34 ${ }^{+}$ progenitors able to promote cancer progression. Cancer Res. 72(1), 325-334 (2012).

54 Hillner BE, Smith TJ. Do the large benefits justify the large costs of adjuvant breast cancer trastuzumab? J. Clin. Oncol. 25(6), 611-613 (2007).

55 Bird BR, Swain SM. Cardiac toxicity in breast cancer survivors: review of potential cardiac problems. Clin. Cancer Res. 14(1), 14-24 (2008).

56 Vaz-Luis I, Keating NL, Lin NU, Lii H, Winer EP, Freedman RA. Duration and toxicity of adjuvant trastuzumab in older patients with early-stage breast cancer: a population-based study. J. Clin. Oncol. 32(9), 927-934 (2014).

57 Reeder-Hayes K, Peacock Hinton S, Meng K, Carey LA, Dusetzina SB. Disparities in use of human epidermal growth hormone receptor 2-targeted therapy for early-stage breast cancer. J. Clin. Oncol. 34(17), 2003-2009 (2016).

58 Baselga J, Cortés J, Kim SB et al.; CLEOPATRA Study Group. Pertuzumab plus trastuzumab plus docetaxel for metastatic breast cancer. N. Engl. J. Med. 366(2), 109-119 (2012).

59 Swain SM, Baselga J, Kim SB et al.; CLEOPATRA Study Group. Pertuzumab, trastuzumab, and docetaxel in HER2-positive metastatic breast cancer. N. Engl. J. Med. 372(8), 724-734 (2015).

60 Miles D, Baselga J, Amadori D et al. Treatment of older patients with HER2-positive metastatic breast cancer with pertuzumab, trastuzumab, and docetaxel: subgroup analyses from a randomized, double-blind, placebo-controlled Phase III trial (CLEOPATRA). Breast Cancer Res. Treat. 142(1), 89-99 (2013).

61 Gianni L, Pienkowski T, Im YH et al. Efficacy and safety of neoadjuvant pertuzumab and trastuzumab in women with locally advanced, inflammatory, or early HER2-positive breast cancer (NeoSphere): a randomised multicentre, open-label, Phase II trial. Lancet Oncol. 13(1), 25-32 (2012).

62 Perez EA, Barrios C, Eiermann W et al. Trastuzumab emtansine with or without pertuzumab versus trastuzumab plus taxane for human epidermal growth factor receptor 2-positive, advanced breast cancer: primary results from the Phase III MARIANNE Study. J. Clin. Oncol. 35(2), 141-148 (2017).

63 The ASCO Post. Phase III APHINITY Study: adjuvant pertuzumab/trastuzumab/chemotherapy increased invasive disease-free survival in HER2-positive breast cancer. www.ascopost.com/News/49404 